\title{
Intenção de recompra no contexto de compras on-line
}

\author{
Alex Eckert ${ }^{I}$ \\ http://orcid.org/0000-0002-8704-9549 \\ Gabriel Sperandio Milan ${ }^{I I}$ \\ http://orcid.org/0000-0003-3480-2653 \\ Deonir de Toni ${ }^{I I I}$ \\ http://orcid.org/0000-0001-9637-8099 \\ I Universidade de Caxias do Sul, Caxias do Sul, RS, Brasil. \\ Doutor em Administração. \\ II Universidade do Vale do Rio dos Sinos, São Leopoldo, RS, Brasil. \\ Doutor em Engenharia da Produção \\ III Universidade de Caxias do Sul, Caxias do Sul, RS, Brasil. \\ Doutor em Administração.
}

http://dx.doi.org/10.1590/1981-5344/3311

O presente estudo teve como objetivo analisar as relações entre os construtos segurança, privacidade, qualidade das informações, confiança e propaganda boca a boca eletrônica (e-WOM) positiva como antecedentes da intenção de recompra dos consumidores no contexto de compras on-line. Para atingir este objetivo, elaborou-se um modelo teórico, seguido de uma survey com uma amostra de 410 consumidores que já realizaram compras on-line, sendo os dados analisados pela Modelagem de Equações Estruturais. Os principais resultados indicam que a segurança, a privacidade e a qualidade das informações do site de compras on-line afeta positivamente a confiança depositada neste site, e que a confiança, por sua vez, impacta positivamente na intenção de recompra dos consumidores. Como implicação destes achados, o estudo contribuiu para uma melhor compreensão acerca dos fatores antecedentes da intenção de recompra dos consumidores que compram no ambiente on-line, o que pode repercutir no sucesso para organizações envolvidas no e-commerce. 
PALAVRAS-CHAVE: comércio eletrônico. Segurança. Privacidade. qualidade das informações. Confiança. intenção de recompra.

\section{Repurchase intentions in the online shopping context}

This study aimed to analyze the relationships between the constructs security, privacy, information quality, trust and positive electronic word-of-mouth (e-WOM) as antecedents of consumers repurchase intention in online shopping context. To achieve this goal, a survey was conducted with a sample of 410 consumers who have already performed this type of operation. The data was statistically analyzed using the Structural Equation Modeling. The main results indicate that security, privacy and the quality of online shopping site information positively affects trust in that site, and that trust, in turn, positively impacts consumers repurchase intention. As implication of these findings, this study contributed to a better understanding of the antecedents of consumers repurchase intention in online shopping context, which can have impact on the success of companies operating their business on the e-commerce.

KEYWORDS: e-commerce. Security. Privacy. Information quality. Trust. Positive electronic word-of-mouth. Repurchase intention.

Recebido em 16.10.2017 Aceito em 17.10.2019

\section{Introdução}

Em meados da década de 1970, Isaac Asimov preconizava que lá pelo ano de 2025 surgiriam lojas de conveniência computadorizadas, nas quais os consumidores fariam suas próprias compras, sem contato físico com vendedores (PETERSON; BALASUBRAMANIAN; BRONNENBERG, 1997). Isto ocorreu muitos anos antes, tendo como um dos principais meios de aceleração deste processo a popularização da Internet (REHMAN, 2012). A partir da efetiva comercialização da Internet para a população na década de 1990, um novo modo de realizar transações comerciais por meio deste canal emergiu na economia mundial: a opção de comprar e de vender produtos e/ou serviços em um ambiente virtual diretamente ao cliente ou consumidor (VAITHIANATHAN, 2010).

As empresas e os consumidores que optaram em realizar suas operações on-line passaram a ter um canal mais rápido de compra, pelo 
qual poderiam interagir sem restrições geográficas (ADNAN, 2014). Mundialmente, as compras on-line do tipo Business-to-Consumer (B2C) vêm crescendo rapidamente, aumentando sua participação no mercado varejista como um todo (RAFFAELE; ORLANDO, 2014), projetando-se que as vendas nesta modalidade devem duplicar no quadriênio 2013-2018 (STATISTA, 2016). Nos últimos quatro anos, as empresas brasileiras de varejo on-line vêm aumentando seu faturamento em $20 \%$ ao ano (EBITEMPRESA, 2015; ECOMMERCENEWS, 2016). Em 2018, o comércio eletrônico no Brasil cresceu 15\%, indicando uma tendência de crescimento de $19 \%$ para 2019 , ou seja, apontando para um crescimento de $\mathrm{R} \$ 79,9$ bilhões (ABCOMM, 2019).

Todavia, a impossibilidade de ter um contato físico com o produto que está sendo adquirido e a falta de contato humano com os vendedores são aspectos que podem inibir a concretização da negociação on-line (VENKATESH, 1998). Nas compras on-line, praticamente toda a relação entre as partes é realizada pelo site específico para tal, cujas características podem interferir nos elementos subjetivos e objetivos que influenciarão o processo de decisão de compra (JOIA; OLIVEIRA, 2008). O que se percebe é que muitos sites não estão preparados para atender todos os requisitos do consumidor para a concretização da compra (BANSAL; ZAHEDI, 2014; KIM; FERRIN; RAO, 2008).

Aspectos como a qualidade das informações do site (BAI; LAW; WEN, 2008; DEDEKE, 2016), a questão da privacidade atinente às informações do consumidor (GUO, 2012) e os riscos sobre a segurança da informação transmitida (ROCA; GARCÍA; VEGA, 2009) podem interferir na decisão de compra. Se tais questões forem percebidas positivamente pelos consumidores, aumentará a confiança depositada no site e a sua vontade de confiar o suficiente para se envolver em uma transação (PONTE; CARVAJAL-TRUJILLO; ESCOBAR-RODRÍGUEZ,2015), potencializando que venham a realizar novas operações (FANG et. al, 2014). No ambiente on-line, a propaganda boca a boca também pode impactar sobremaneira na intenção de (re)compra dos consumidores (ESTEVES, 2014).

Pesquisas apontam que a intenção de recompra é influenciada por estes diferentes construtos, mas que há uma carência de estudos mais elucidativos (GEFEN; BENBASAT; PAVLOU, 2008; CHEN et. al, 2016; TEHREEM, 2017). Estes construtos são, frequentemente, analisados de forma isolada, não se importando com o contexto em que as pesquisas foram realizadas, tampouco acerca do perfil dos consumidores pesquisados (HA; JANDA; MUTHALY, 2010). A questão geográfica, contemplando características culturais e socioeconômicas dos consumidores, é outro aspecto que justifica a realização da presente pesquisa no contexto brasileiro, pois ainda são restritas as pesquisas em países em desenvolvimento (ADNAN, 2014) ou na América Latina (BIANCHI; ANDREWS, 2012).

Diante deste contexto, sua relevância e justificativas apresentadas, é necessário que se tenha uma melhor compreensão acerca das variáveis atinentes ao contexto das compras on-line. Para tanto, a presente 
pesquisa teve como objetivo analisar as relações entre os construtos segurança, privacidade, qualidade das informações, confiança e propaganda boca a boca eletrônica positiva (e-WOM) como antecedentes da intenção de recompra dos consumidores no contexto de compras online.

\section{Referencial teórico, hipóteses de pesquisa e modelo teórico proposto}

\subsection{Confiança}

A evolução histórica fez com que a confiança tivesse diferentes conceitos. Todavia, dois enfoques permearam a pesquisa e o pensamento sobre a confiança. Ela implica na assunção de riscos e algum tipo de confiança é inerente a todos os relacionamentos (SHEPPARD; SHERMANN, 1998). Trata-se de uma expectativa positiva em uma situação de risco futuro, em que as vulnerabilidades de uma das partes não vão ser exploradas pela outra parte envolvida (CORRITORE; KRACHER; WIEDENBECK, 2003). A confiança é considerada como um elemento essencial no contexto das compras on-line (GAO et.al, 2002). Precisa-se de confiança no ambiente on-line quando dos dados pessoais e informações financeiras são compartilhadas ao efetivar a transação (EGGERT, 2006). Em uma visão mais extrema, pode-se afirmar que a efetivação de compras on-line depende da confiança do consumidor em relação à empresa ou ao seu site (WANG; EMURIAN, 2005).

Estudos envolvendo a confiança foram realizados no contexto de compras on-line (URBAN; AMYX; LORENZON, 2009). Aliás, a confiança online tem sido a influência predominante na intenção de (re)compra on-line (HSU; CHANG; CHUANG, 2015). Ponte, Carvajal-Trujillo e EscobarRodríguez (2015) analisaram a influência da confiança percebida na intenção de utilizar sites de compras on-line, especificamente com serviços de viagem, evidenciando que a confiança é um preditor da intenção de compras on-line. Já Fang e outros (2014) investigaram a relação entre confiança e recompra on-line, e concluíram que há relação entre os dois construtos.

\subsection{Segurança do site}

No momento em que o consumidor adquire produtos on-line, pode haver uma percepção de risco envolvida na transmissão de informações pessoais pela Internet. Devido a isso, a compra de produtos pela Internet envolve a segurança percebida como um aspecto fundamental (SALISBURY et.al, 2001). Segurança percebida pode ser definida como a probabilidade subjetiva em que os consumidores acreditam que sua informação pessoal não será vista, armazenada e manipulada inadequadamente pela empresa com a qual estão comprando (FLAVIÁN; GUINALÍU, 2006). 
A ameaça de segurança no ambiente virtual pode ser qualquer circunstância, condição ou evento com potencial de causar prejuízos econômicos, ou exposição do consumidor, mediante acesso indevido a informações pessoais, mediante divulgação, alteração de conteúdos, fraude ou abuso (CHOU; CHEN; LIN, 2015). Para atender aos requisitos de segurança no e-commerce, devem ser utilizadas tecnologias e protocolos para deixar as operações mais seguras. A minimização dos riscos envolvidos nestas operações e o consequente aumento da segurança podem ser proporcionados pela criptografia, certificação e assinaturas digitais (GARFIELD; MCKEOWN, 1997). A segurança percebida é reconhecida como um antecedente-chave para a formação da confiança dos consumidores (RAY; OW; KIM, 2011; PANDA, S.; PANDA, J., 2012), relacionando-se de forma consistente com as suas expectativas de confiança em relação aos sites ou empresas no ambiente virtual (FLAVIÁN; GUINALÍU, 2006).

Requisitos de controle da segurança on-line foram identificados como formadores da confiança dos consumidores (BANSAL; ZAHEDI, 2014), e garantias de segurança disponibilizadas por varejistas on-line são um meio de estimular a confiança inicial dos consumidores (SCHLOSSER; WHITE; LLOYD, 2006; CHOI; NAZARETH, 2014). Sendo assim, é apresentada a primeira hipótese de pesquisa:

H1: A segurança percebida pelos consumidores influencia positivamente a confiança depositada no site de compras on-line.

\subsection{A privacidade}

A proteção da privacidade para transações nas compras on-line é muito relevante para as empresas que atuam neste mercado (GUO, 2012). A privacidade do consumidor compreende a preocupação com o processo de coleta de dados e informações e pelo uso inadequado, ou não, destes conteúdos (CASTAÑEDA; MONTOSO; LUQUE, 2007). A privacidade percebida, então, é a capacidade dos consumidores controlarem a presença de outras pessoas ou agentes intervenientes no ambiente virtual durante uma determinada transação, tendo a exata noção sobre a divulgação destes conteúdos a terceiros (MEZIANE; KASIRAN, 2008; ANTONIOU; BATTEN, 2011).

Nas compras on-line, a privacidade está direcionada à preocupação com a informação que é gerada como resultado final das ações dos consumidores. Neste contexto, lida-se com atividades on-line dos consumidores e consiste da coleta de dados e informações, do seu compartilhamento, utilização e/ou reutilização e armazenamento adequado de registros atinentes às transações efetivadas (ALHARBI; ZYNGIER; HODKINSON, 2013; DINEV, 2014).

A privacidade das informações influencia a confiança depositada pelo consumidor no site de compras on-line. Quando os sites se preocupam com a privacidade do consumidor existe uma maior confiança deste em relação ao processo de comprar on-line (CHELLAPPA; PAVLOU, 2002; METZGER, 2004; BART et.al, 2005). A percepção de privacidade dos consumidores tem um efeito positivo sobre a construção e manutenção da 
sua confiança em um referido site (LIU et.al, 2005; ROMAN, 2007). As práticas de privacidade dos sites reduzem o risco e aumentam a confiança dos consumidores em relação ao site (VAN SLYKE et.al, 2006; D'ALESSANDRO; GIRARDI; TIANGSOONGNERN, 2012). Por conseguinte, emerge a segunda hipótese de pesquisa:

H2: A privacidade percebida pelos consumidores influencia positivamente a confiança depositada no site de compras on-line.

\subsection{Qualidade do site e das informações disponibilizadas}

A qualidade do site de compras on-line influencia a decisão de compra dos consumidores (SHCHIGLIK; BARNES, 2004) e a sua disposição em transacionar com determinada empresa (PARK; LEE; WIDDOWS, 2004), inclusive de forma sistemática (HUANG, 2008; SRINIVASAN; ANDERSON; PONNAVOLU, 2002). Diante disso, percebe-se que o sucesso de empresas de e-commerce está fortemente associado à qualidade de seu site (KAYA, 2010).

Quando se aborda a qualidade dos sites, é necessário considerar que é algo complexo (ALADWANI; PALVIA, 2002). Para verificar a qualidade do site, o consumidor geralmente utiliza a qualidade das informações disponíveis, configurando-se como a percepção geral dos consumidores sobre a integridade e a exatidão das informações constantes no site, relativas tanto às ofertas quanto aos procedimentos para a realização de uma operação de compra on-line (KIM; FERRIN; RAO, 2008). A qualidade da informação do site é vital para o sucesso dos empreendimentos on-line (MCKINNEY; YOON; ZAHEDI, 2002; DELONE; MCLEAN, 2003).

Em relação à qualidade dos sites de compras on-line e a confiança depositada pelos compradores nestes sites, há evidências de que a qualidade do conteúdo do site é um antecedente da confiança do consumidor (MCKNIGHT; KACMAR; CHOUDHURY, 2004). Kim, Ferrin e Rao (2008) confirmaram tal relação, convergindo com os resultados encontrados por Ponte, Carvajal-Trujillo e Escobar-Rodríguez (2015), os quais verificaram que o principal determinante da confiança é a qualidade da informação. Outros estudos indicam que a qualidade das informações disponibilizadas pelos sites tem um impacto, na maioria das vezes, significativo sobre a confiança do consumidor no site (MCKNIGHT; KACMAR; CHOUDHURY, 2004; SHCHIGLIK; BARNES, 2004). Com base nisso, formulou-se a terceira hipótese de pesquisa:

H3: A qualidade das informações percebida pelos consumidores influencia positivamente a confiança depositada no site de compras online.

Quando o assunto é o comportamento do consumidor, existem determinadas características que podem interferir nas suas decisões e percepções acerca de um processo de compra (DOMMEYER; GROSS, 2003). Aspectos como experiência e frequência com que os consumidores realizam compras on-line podem afetar percepções e decisões de compra (BERNARD; MAKIENKO, 2011; KHALIFA; LIU, 2007). Com base nisso, é possível supor que a frequência de compras na Internet interfira (efeito 
moderador) na relação entre a qualidade das informações percebida pelos consumidores e a confiança depositada no site de compras on-line.

\subsection{Propaganda boca a boca eletrônica}

A propaganda boca a boca word-of-mouth (WOM), caracterizada pela troca de informações entre as pessoas sobre empresas, marcas, produtos e/ou serviços (SCHÜTZE, 2014), tornou-se um tema relevante, pela sua eficácia como elemento de comunicação (TRUSOV; BUCKLIN; PAUWELS, 2009). Os efeitos da WOM foram sendo potencializados pelo avanço de novas tecnologias, principalmente pela disseminação da Internet (CHEUNG; THADANI, 2012), emergindo neste novo cenário a propaganda boca a boca eletrônica eletronic word-of-mouth (e-WOW), que diferentemente da WOM tradicional, pode ser disponibilizada para uma quantidade imensa de pessoas e organizações por meio da Internet (HENNIG-THURAU et.al, 2004).

Com o avanço das tecnologias, os consumidores têm a possibilidade de compartilhar informações relacionadas às empresas, marcas e suas ofertas por meio da Internet, aumentando o potencial impacto da WOM (CHEUNG; THADANI, 2012). Neste ambiente, a e-WOM é favorecida. Aliás, a e-WOM pode ser definida como qualquer comentário ou declaração positiva ou negativa feita por potenciais, atuais ou antigos consumidores sobre uma determinada empresa, marca, produto e/ou serviço disponibilizado para uma multidão de pessoas e organizações no ambiente virtual (HENNIG-THURAU et.al, 2004). Pesquisadores reconheceram a importância crescente da e-WOM nas operações on-line devido ao seu forte crescimento nos últimos anos (ABRANTES et.al, 2013), pois é uma forma de comunicação poderosa para as compras online (YOO; SANDERS; MOON, 2013; SCHÜTZE, 2014), potencializada pelas redes que se formam na Internet (TENG et.al, 2014).

Por isso, a confiança é um componente crítico para a troca de informações com outros membros em uma rede social como em outros ambientes da Internet, podendo motivar comportamentos nos receptores das informações (CHU; KIM, 2011). No ambiente on-line, ela propicia o compartilhamento de informações pessoais e ações específicas resultantes de conselhos recebidos ou pela troca de experiências (MCKNIGHT; KACMAR; CHOUDHURY, 2004). Assim, a confiança pode estar relacionada à e-WOM à medida que leva os consumidores a compartilharem informações pessoais, fazer compras on-line e dar conselhos a outros (HSIAO et.al, 2010). Ou seja, a confiança tende a fazer com que os consumidores compartilhem informações pessoais de maneira mais confortável e efetiva (LEE; KANG; MCKNIGHT, 2007). Por isso, propõe-se a quarta hipótese de pesquisa:

H4: A confiança dos consumidores no site de compras on-line influencia positivamente no seu envolvimento em propaganda boca a boca eletrônica (e-WOM) positiva. 


\subsection{Intenção de recompra}

A repetição de compras pelos consumidores é a principal fonte de sustentação de um bom volume de negócios para as empresas (GUPTA; KIM, 2007; LU; LU; WANG, 2012), pelo fato do retorno líquido sobre os investimentos ser muito maior para as estratégias de retenção do que para as de atração ou conquista de novos clientes (BOJEI et.al, 2013). Portanto, a repetição de compras, ligada diretamente à retenção de clientes, tornou-se uma questão essencial para os vendedores on-line (SHIN et.al, 2013; TSAO, 2013). A intenção de recompra é a probabilidade do consumidor de se engajar em um comportamento de repetição de compras futuras (VALLEJO; REDONDO; ACERETE, 2015) e, nas compras on-line, remete-se à probabilidade subjetiva de que um indivíduo irá continuar comprando os produtos do mesmo site (CHIU et.al, 2009; LIN; WANG, 2015).

Estudos relacionaram a confiança à intenção de recompra, identificando que a confiança é um antecedente da intenção de recompra (QURESHI et.al, 2009; CHIU et.al, 2014), tendo efeito positivo sobre esta (CASALÓ; FLAVIÁN; GUINALÍU, 2010; LIN; WANG, 2015). Resultados semelhantes foram encontrados por Chiu e outros (2009) e Hsu, Chang e Chuang (2015), que encontraram evidências de que a confiança é um preditor da intenção de recompra dos consumidores nos sites de compras on-line. Assim, elaborou-se a quinta hipótese de pesquisa:

H5: A confiança dos consumidores influencia positivamente a sua intenção de recompra no site de compras on-line.

Outras pesquisas buscaram analisar as relações existentes entre a e-WOM e a intenção de recompra dos consumidores. Para Stauss (1997), a e-WOM tem um forte impacto sobre as intenções e as decisões de compra dos consumidores e é um fator chave no mercado de compras online. López e Sicilia (2014) argumentam que as empresas que operam no ambiente on-line devem ter em mente que a e-WOM tem um grande impacto no comportamento do consumidor, fato sustentado pela evidência de que muitas delas tentam estimular e gerenciar este comportamento.

Foram encontraram evidências de impactos positivos entre a e-WOM e as vendas de produtos no comércio eletrônico (CHEVALIER; MAYZLIN, 2006; DELLAROCAS; AWAD; ZHANG, 2007). Verificou-se, ainda, a influência da e-WOM nas motivações e comportamentos dos compradores em realizar e repetir operações comerciais (CHEUNG; LEE, 2012; LÓPEZ; SICILIA, 2014). Formulou-se, então, a sexta hipótese de pesquisa:

H6: A propaganda boca a boca eletrônica (e-WOM) positiva influencia positivamente a intenção de recompra do consumidor no site de compras on-line.

A partir dos argumentos apresentados, a Figura 1 traz o Modelo Teórico proposto, bem como suas respectivas hipóteses de pesquisa. 
Figura 1 - Modelo Teórico proposto

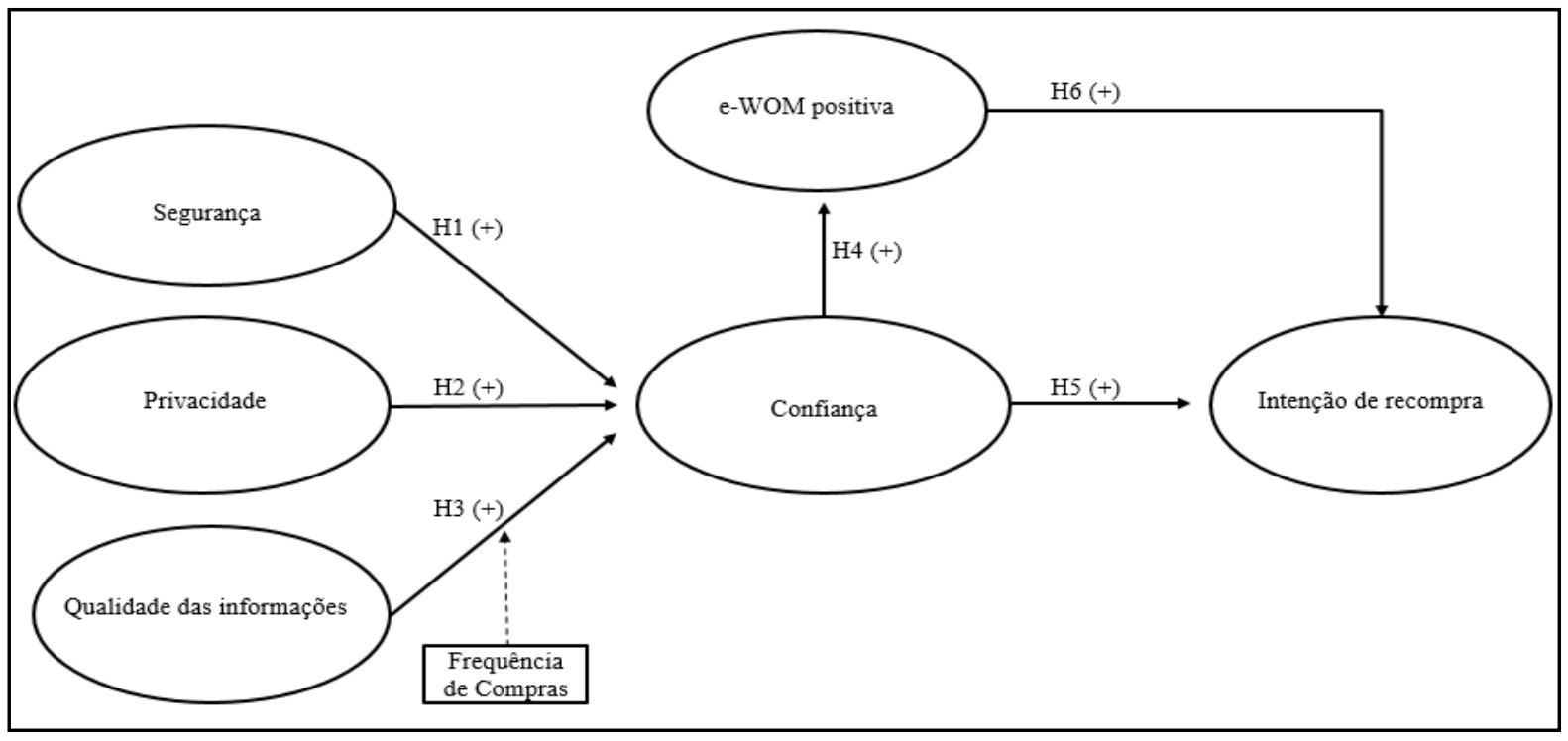

Fonte: produção dos autores.

\section{Aspectos metodológicos}

Procedeu-se a uma pesquisa do tipo survey, de corte transversal, com 424 consumidores, que já haviam realizado compras on-line. Para tanto, foi elaborado um questionário de pesquisa que serviu como instrumento para a coleta de dados. Para aferição dos construtos foi utilizada uma escala intervalar do tipo Likert de sete pontos ("1. Discordo Totalmente" a "7. Concordo Totalmente").

$\mathrm{Na}$ operacionalização dos construtos, foram adotadas as seguintes escalas: Segurança do Site, escala de cinco itens (ESCOBARRODRÍGUEZ; CARVAJAL-TRUJILLO, 2014); Privacidade do Site, escala de sete itens (FLAVIÁN; GUINALÍU, 2006); Qualidade das Informações, escala de sete itens (KUAN; BOCK; VATHANOPHAS, 2008); Confiança no Site, escala de seis itens (CHANG; CHEN, 2008); e-WOM Positiva, escala de seis itens (CHU; KIM, 2011); e Intenção de Recompra, escala de três itens (HSU; CHANG; CHUANG, 2015). Estruturado o questionário, procedeu-se à validação de face, submetendo-o a três professores pesquisadores, experts da área e, logo após, ao seu pré-teste, com vinte respondentes com perfil semelhante à população-alvo. Tais questionários não foram incorporados à amostra final.

A coleta de dados para o desenvolvimento da pesquisa compreendeu a entrega dos questionários diretamente pelos pesquisadores aos possíveis respondentes, aguardando o preenchimento para após fazer o recolhimento dos mesmos. O processamento dos questionários foi realizado à medida que estes iam sendo aplicados. Cada questionário recebeu um código, sendo inspecionados e tabulados no banco de dados. A análise dos dados foi realizada utilizando-se estatística 
multivariada de dados, tomando-se por base a técnica de Modelagem de Equações Estruturais.

A amostra atendeu às suposições de normalidade, homoscedasticidade, linearidade e multicolinearidade dos dados e após o tratamento de missings e outliers (uni e multivariados) (HAIR Jr. et.al, 2010) resultou em uma amostra final de 410 casos. A matriz de entrada de dados escolhida foi a matriz de covariância e o método de estimação o da Máxima Verossimilhança (KLINE, 2011).

\section{Resultados da pesquisa}

\subsection{Perfil da amostra}

Quanto ao perfil da amostra, composta por conveniência, e por consumidores da Serra Gaúcha, cabe comentar que 252 (61,5\%) dos respondentes são do gênero feminino e 158 (38,5\%) do masculino, sendo 132 (32,2\%) deles com faixa etária entre 17 a 21 anos, $192(46,8 \%)$ entre 22 a 26 anos e $86(21,0 \%)$ entre 27 a 49 anos. A maioria deles (384 ou $93,7 \%$ ) tem renda individual mensal até $\mathrm{R} \$ 3.940,00$, gasta anualmente entre $R \$ 100,01$ a $R \$ 1.999,00$ (301 ou 73,4\%), com 3 a 6 compras anuais (241 ou 58,8\%). Os sites de compra preferidos são Mercado Livre (59 ou 14,4\%), Netshoes (51 ou 12,4\%), Submarino (44 ou 10,7\%), Americanas (40 ou 9,8\%), Magazine Luiza (28 ou 6,8\%) e Saraiva (26 ou 6,3\%). Os produtos mais comprados são roupas e calçados (216 ou 52,7\%), equipamentos eletrônicos (215 ou $52,4 \%$ ) e livros e revistas (149 ou $36,3 \%)$.

\subsection{Validação individual dos construtos}

Para a análise da unidimensionalidade, foi realizada a Análise Fatorial Exploratória, pelo método de componentes principais através da rotação ortogonal Varimax para cada um dos construtos, por meio da maximização do quadrado das variâncias das cargas dos fatores (MALHOTRA; BIRKS; WILLS, 2012). Foram encontradas cargas fatoriais muito boas em praticamente todas as variáveis, ou seja, acima de 0,5. A exceção foram duas variáveis que apresentaram cargas de 0,459 e 0,433, que, mesmo inferiores às demais, são consideradas aceitáveis (HAIR Jr. et.al, 2010). Em complemento, foram realizados os testes de Esfericidade de Bartlett e o de Kaiser-Meyer-Olkin (KMO). O primeiro deles indicou que as correlações foram significativas ao nível 0,000 e o outro apurou um valor de 0,936. Os resultados aderem às recomendações (HAIR Jr. et.al, 2010; MALHOTRA; BIRKS; WILLS, 2012).

A confiabilidade foi verificada através do Alpha de Cronbach, indicando que todos estão acima dos valores recomendados, e das variâncias explicadas, que ficaram acima de 0,5, que é o valor mínimo recomendado (HAIR Jr. et.al, 2010). A Tabela 1 traz um resumo destes resultados. 
Tabela 1 - Cargas fatoriais, variância explicada e Alpha de Cronbach

\begin{tabular}{|c|c|c|c|c|}
\hline CONSTRUTOS & VARIÁVEIS & $\begin{array}{c}\text { CARGAS } \\
\text { FATORIAIS }\end{array}$ & $\begin{array}{l}\text { VARIÂNCIA } \\
\text { EXPLICADA }\end{array}$ & $\begin{array}{l}\text { ALPHA DE } \\
\text { CRONBACH }\end{array}$ \\
\hline \multirow{5}{*}{ Segurança } & SEGUR_1 & 0,725 & \multirow{5}{*}{$60,30 \%$} & \multirow{5}{*}{0,820} \\
\hline & SEGUR_2 & 0,700 & & \\
\hline & SEGUR_3 & 0,715 & & \\
\hline & SEGUR_4 & 0,574 & & \\
\hline & SEGUR_5 & 0,671 & & \\
\hline \multirow{7}{*}{ Privacidade } & PRIV_1 & 0,652 & \multirow{7}{*}{$57,40 \%$} & \multirow{7}{*}{0,843} \\
\hline & PRIV_2 & 0,604 & & \\
\hline & PRIV_3 & 0,676 & & \\
\hline & PRIV_4 & 0,750 & & \\
\hline & PRIV_5 & 0,753 & & \\
\hline & PRIV_6 & 0,755 & & \\
\hline & PRIV_7 & $0,459 *$ & & \\
\hline \multirow{7}{*}{$\begin{array}{l}\text { Qualidade } \\
\text { das } \\
\text { Informações }\end{array}$} & QUAL_INF_1 & 0,773 & \multirow{7}{*}{$61,07 \%$} & \multirow{7}{*}{0,866} \\
\hline & QUAL_INF_2 & 0,777 & & \\
\hline & QUAL_INF_3 & 0,779 & & \\
\hline & QUAL_INF_4 & 0,727 & & \\
\hline & QUAL_INF_5 & 0,705 & & \\
\hline & QUAL_INF_6 & $0,433 *$ & & \\
\hline & QUAL_INF_7 & 0,590 & & \\
\hline \multirow{6}{*}{ Confiança } & CONF_S_1 & 0,554 & \multirow{6}{*}{$74,17 \%$} & \multirow{6}{*}{0,930} \\
\hline & CONF_S_2 & 0,672 & & \\
\hline & CONF_S_3 & 0,635 & & \\
\hline & CONF_S_4 & 0,675 & & \\
\hline & CONF_S_5 & 0,700 & & \\
\hline & CONF_S_6 & 0,706 & & \\
\hline \multirow{6}{*}{$\begin{array}{l}\text { e-WOM } \\
\text { Positiva }\end{array}$} & EWOM_1 & 0,811 & \multirow{6}{*}{$71,68 \%$} & \multirow{6}{*}{0,920} \\
\hline & EWOM_2 & 0,836 & & \\
\hline & EWOM_3 & 0,885 & & \\
\hline & EWOM_4 & 0,822 & & \\
\hline & EWOM_5 & 0,875 & & \\
\hline & EWOM_6 & 0,838 & & \\
\hline \multirow{3}{*}{$\begin{array}{l}\text { Intenção de } \\
\text { Recompra }\end{array}$} & INT_REC_1 & 0,739 & \multirow{3}{*}{$89,43 \%$} & \multirow{3}{*}{0,940} \\
\hline & INT_REC_2 & 0,757 & & \\
\hline & INT_REC_3 & 0,712 & & \\
\hline
\end{tabular}

Obs.: $\left(^{*}\right)$ Cargas fatoriais $<0,5$, mas $>0,4$.

Fonte: produção dos autores

Em termos da validade convergente, foram avaliadas a confiabilidade composta e a variância extraída de cada um dos construtos (FORNELL; LARCKER, 1981). Conforme a Tabela 2, os valores da confiabilidade composta e variância extraída para todos os construtos são aceitáveis. Todos os valores são superiores ao recomendado (HAIR Jr. et.al, 2010; MALHOTRA; BIRKS; WILLS, 2012). 
Tabela 2 - Confiabilidade composta e variância extraída

\begin{tabular}{c|c|c}
\hline CONSTRUTOS & $\begin{array}{c}\text { CONFIABILIDADE } \\
\text { COMPOSTA }\end{array}$ & VARIÂNCIA EXTRAÍDA \\
\hline Segurança & 0,835 & $50,83 \%$ \\
\hline Privacidade & 0,875 & $51,10 \%$ \\
\hline Qualidade das Informações & 0,892 & $55,43 \%$ \\
\hline Confiança & 0,930 & $69,03 \%$ \\
\hline e-WOM Positiva & 0,921 & $66,13 \%$ \\
\hline Intenção de Recompra & 0,942 & $84,40 \%$ \\
\hline
\end{tabular}

Fonte: produção dos autores.

Por fim, analisou-se a validade discriminante entre os construtos pelo procedimento de Fornell e Larcker (1981), pelo qual as variâncias extraídas dos construtos são comparadas com as variâncias compartilhadas, obtidas a partir das correlações entre construtos elevadas ao quadrado. Em quase todas as comparações há validade discriminante. Porém, em duas situações isto não ocorreu (Tabela 3).

Tabela 3 - Validade discriminante pelo critério de Fornell e Larcker

\begin{tabular}{c|c|c|c|c|c|c}
\hline CONSTRUTOS & SEGUR & PRIV & QUAL_INF & CONF & $\begin{array}{c}\text { E- } \\
\text { WOM }\end{array}$ & INT_REC \\
\hline Segurança & $\mathbf{0 , 5 0 8}$ & & & & & \\
\hline Privacidade & 0,450 & $\mathbf{0 , 5 1 1}$ & & & & \\
\hline $\begin{array}{c}\text { Qualidade das } \\
\text { Informações }\end{array}$ & 0,407 & 0,378 & $\mathbf{0 , 5 5 4}$ & & & \\
\hline Confiança & 0,442 & 0,546 & 0,590 & $\mathbf{0 , 6 9 0}$ & & \\
\hline e-WOM Positiva & 0,003 & 0,008 & 0,000 & 0,007 & $\mathbf{0 , 6 6 1}$ & \\
\hline Intenção de Recompra & 0,372 & 0,291 & 0,376 & 0,494 & 0,008 & $\mathbf{0 , 8 4 4}$ \\
\hline
\end{tabular}

Obs.: Os valores negritados demonstram as variâncias extraídas e os demais as variâncias compartilhadas

Fonte: produção dos autores.

Nas situações em que a validade discriminante não é satisfatória, é recomendada a realização de outro teste, o de Bagozzi e Phillips (1982). Procedeu-se, então, à verificação da validade discriminante entre a privacidade e a confiança e entre a qualidade das informações e a confiança (Tabela 4). 
Tabela 4 - Validade discriminante pelo critério de Bagozzi e Phillips

\begin{tabular}{c|c|c|c|c|c}
\hline $\begin{array}{c}\text { CONSTRUTO } \\
\mathbf{1}\end{array}$ & $\begin{array}{c}\text { CONSTRUTO } \\
\mathbf{2}\end{array}$ & $\begin{array}{c}\chi^{\mathbf{2}} \text { (MODELO } \\
\text { FIXO) }\end{array}$ & $\begin{array}{c}\chi^{\mathbf{2}} \text { (MODELO } \\
\text { LIVRE) }\end{array}$ & DIF. & SIG. \\
\hline Privacidade & Confiança & 135,080 & 106,696 & 28,384 & 0,000 \\
\hline $\begin{array}{c}\text { Qualidade } \\
\text { das } \\
\text { Informações }\end{array}$ & Confiança & 104,811 & 69,222 & 35,589 & 0,000 \\
\hline
\end{tabular}

Fonte: produção dos autores.

Com base nos resultados, nas duas situações, os valores das diferenças entre $0 \chi^{2}$ do modelo fixo e do modelo livre são significativos, indicando que não existe correlação entre os construtos. Sendo os valores considerados aceitáveis, assume-se a validade discriminante dos construtos.

\subsection{Validação do modelo teórico}

A validação do Modelo Teórico foi realizada inicialmente pela a análise da qualidade das medidas de ajuste do modelo, cujos resultados constam da Tabela 5.

Tabela 5 - Medidas de ajuste do Modelo Teórico

\begin{tabular}{c|c|c}
\hline $\begin{array}{c}\text { MEDIDAS DE } \\
\text { AJUSTE }\end{array}$ & RECOMENDADO & VALORES \\
\hline GFI & $>0,90$ & 0,865 \\
\hline AGFI & $>0,90$ & 0,836 \\
\hline RMSEA & $>0,05 \mathrm{e}<0,08$ & 0,056 \\
\hline CFI & $>0,90$ & 0,937 \\
\hline TLI & $>0,90$ & 0,928 \\
\hline NFI & $>0,90$ & 0,900 \\
\hline
\end{tabular}

Fonte: produção dos autores.

Os resultados foram satisfatórios (KLINE, 2011; ARBUCKLE; 2016; BYRNE, 2016). Apenas dois índices ficaram abaixo do recomendado pela literatura (GFI, 0,865 e AGFI, 0,836). Porém, valores de GFI e AGFI podem variar muito em função do tamanho da amostra e simulações apontam que tais índices não apresentam valores tão significativos quanto os valores encontrados em outras medidas (BAGOZZI; YI, 2012; NUNKOO; RAMKISSOON; GURSOY, 2013), as quais obtiveram resultados satisfatórios para o modelo testado.

Dando continuidade à validação do Modelo Teórico, foi realizado o teste de hipóteses, examinando-se a significância e a magnitude dos coeficientes de regressão estimados, aliás, conforme mostra a Tabela 6. 
Tabela 6 - Teste de hipóteses

\begin{tabular}{|c|c|c|c|c|c|c|c|}
\hline Hi & $\begin{array}{c}\text { CAMINHOS } \\
\text { ESTRUTURAIS }\end{array}$ & $\begin{array}{l}\text { COEFICIENTES } \\
\text { NÃO } \\
\text { PADRONIZADOS } \\
\text { (b) }\end{array}$ & $\begin{array}{c}\text { ERRO } \\
\text { PADRÃO }\end{array}$ & $\begin{array}{c}\text { COEFICIENTES } \\
\text { PADRONIZADOS } \\
(\beta)\end{array}$ & $\begin{array}{c}T- \\
\text { VALUES }\end{array}$ & $\mathbf{P}$ & RESULTADOS \\
\hline $\mathrm{H}_{1}$ & $\begin{array}{l}\text { SEGUR } \square \\
\text { CONF_S }\end{array}$ & 0,164 & 0,040 & 0,223 & 4,124 & $\begin{array}{c}\mathrm{p}< \\
0,001\end{array}$ & Suportada \\
\hline $\mathrm{H}_{2}$ & PRIV $\square$ CONF_S & 0,330 & 0,045 & 0,437 & 7,337 & $\begin{array}{c}p< \\
0,001\end{array}$ & Suportada \\
\hline $\mathrm{H}_{3}$ & $\begin{array}{l}\text { QUAL_INF } \square \\
\text { CONF_S } \\
\end{array}$ & 0,521 & 0,050 & 0,637 & 10,487 & $\begin{array}{c}p< \\
0,001 \\
\end{array}$ & Suportada \\
\hline $\mathrm{H}_{4}$ & $\begin{array}{l}\text { CONF_S } \square \\
\text { EWOM }\end{array}$ & 0,108 & 0,088 & 0,055 & 1,231 & $\begin{array}{c}p= \\
0,218\end{array}$ & Não suportada \\
\hline $\mathrm{H}_{5}$ & $\begin{array}{l}\text { CONF_S } \square \\
\text { INT_REC }\end{array}$ & 0,753 & 0,050 & 0,702 & 15,180 & $\begin{array}{c}\mathrm{p}< \\
0,001\end{array}$ & Suportada \\
\hline $\mathrm{H} 6$ & $\begin{array}{l}\text { EWOM } \square \\
\text { INT_REC }\end{array}$ & 0,019 & 0,024 & 0,034 & 0,763 & $\begin{array}{c}p= \\
0,446\end{array}$ & Não suportada \\
\hline
\end{tabular}

Obs.: Nível de significância de 0,05.

Fonte: produção dos autores.

Quatro das seis hipóteses propostas foram suportadas estatisticamente: H1 $(\beta=0,223, p<0,001)$, H2 $(\beta=0,437, p<0,001)$, H3 $(\beta=0,637, p$ $<0,001)$ e $\mathbf{H 5}(\beta=0,702, p<0,001)$. As outras duas hipóteses, $\mathbf{H}_{4}(\beta=$ $0,055, p=0,218)$ e $\mathbf{H 6}(\beta=0,034, p=0,446)$, não foram suportadas.

Outra forma de verificar a efetividade do teste de hipóteses é por meio dos Coeficientes de Determinação (R2). Quanto maior for o poder de explicação da equação da regressão, melhor será a predição da variável dependente (HAIR Jr. et.al, 2010). Constatou-se que 49,7\% da variância da Intenção de Recompra $\left(R^{2}=0,497\right)$ é explicada por seus antecedentes, contemplados no Modelo Teórico. A Tabela 7 apresenta os resultados.

Tabela 7 - Coeficientes de determinação

\begin{tabular}{c|c}
\hline CONSTRUTOS & $\mathbf{R}^{\mathbf{2}}$ \\
\hline Confiança & 0,646 \\
\hline e-WOM Positiva & 0,003 \\
\hline Intenção de Recompra & 0,497 \\
\hline
\end{tabular}

Fonte: produção dos autores.

\subsection{Teste da moderação da frequência de compras}

Buscando encontrar resultados complementares, foi analisada se a frequência de compras interfere na relação entre a qualidade das informações percebida pelos consumidores e a confiança depositada no site de compras on-line. A literatura sugere que, nestes casos, sejam testados os efeitos de moderação (BARON; KENNY, 1986), sendo empregado o recurso dos Índices Críticos para Diferenças entre os Parâmetros. A Tabela 8 é elucidativa. 
Tabela 8 - Efeito moderador da frequência de compras

\begin{tabular}{|c|c|c|c|c|c|c|c|}
\hline $\begin{array}{c}\text { RELAÇÃ } \\
0\end{array}$ & $\begin{array}{c}\text { COMPR } \\
\text { AS } \\
\text { ANUAIS }\end{array}$ & $\begin{array}{l}\text { COEFICIENTE } \\
\text { S NÃO } \\
\text { PADRONIZAD } \\
\text { OS (b) }\end{array}$ & $\begin{array}{l}\text { ERRO } \\
\text { PADRÃ } \\
0\end{array}$ & $\begin{array}{c}\text { COEFICIENTE } \\
\text { S } \\
\text { PADRONIZAD } \\
\text { OS }(\beta)\end{array}$ & $\begin{array}{c}T- \\
\text { VALUE } \\
\quad S\end{array}$ & $\mathbf{P}$ & $\begin{array}{c}\text { Z } \\
\text { SCOR }\end{array}$ \\
\hline \multirow{2}{*}{$\begin{array}{c}\text { QUAL_I } \\
\text { NF } \\
\square \text { CONF }\end{array}$} & 1 & 0,559 & 0 & 0,658 & 9 & $\begin{array}{c}p<0,00 \\
1\end{array}$ & \multirow{2}{*}{2,315} \\
\hline & 7 ou mais & 0,305 & 0,094 & 0,486 & 3,248 & $\mathrm{p}=0,001$ & \\
\hline
\end{tabular}

Fonte: produção dos autores.

A relação entre os construtos é potencializada junto ao grupo que realizou de 1 a 6 compras nos últimos doze meses, indicando que a qualidade das informações tem uma influência positiva mais forte sobre a confiança depositada no site de compras on-line para consumidores que realizam menos compras se comparados com aqueles que realizaram uma quantidade maior de compras ( 7 ou mais). Tais resultados são semelhantes aos de Kim e Noh (2012), que identificaram o efeito moderador da experiência do consumidor na relação entre a qualidade das informações dos sites e a confiança depositada nestes sites. Portanto, é possível inferir que a qualidade das informações desempenha um papel mais importante sobre a confiança depositada neste site para consumidores que realizam compras on-line com menor frequência.

\section{Considerações finais}

A primeira contribuição do estudo é acerca dos construtos inseridos no Modelo Teórico proposto, em uma tentativa de compreender melhor os antecedentes da intenção de recompra no contexto de compras on-line, o que foi indicado como prioridade de pesquisa por órgãos representativos da área do marketing (MSI, 2015; AMA, 2015), ainda mais pesquisas em países latino-americanos (BIANCHI; ANDREWS, 2012; ADNAN, 2014). A segunda diz respeito ao poder explicativo dos construtos antecedentes da intenção de recompra testados $\left(R^{2}=0,497\right)$. Os resultados indicam que praticamente a metade da sua variância pode ser explicada pela segurança, privacidade, qualidade das informações percebida, e-WOM positiva e confiança depositada no site de compras on-line.

A terceira é a confirmação da H1 (a segurança percebida pelos consumidores influencia positivamente a confiança depositada no site de compras on-line). Os aspectos relativos à segurança percebida pelos consumidores que compram no ambiente on-line estão relacionados de uma forma sólida com as suas expectativas de confiança nos sites das empresas que operam no ambiente virtual. A quarta é a confirmação da H2 (a privacidade percebida pelos consumidores influencia positivamente a confiança depositada no site de compras on-line). Quando os sites manifestam uma preocupação com a privacidade do consumidor, há uma confiança maior deste em relação ao comportamento de concretizar compras on-line. A disponibilização da declaração de privacidade do site 
pode despertar a percepção de que o site oferece um alto grau de privacidade, aumentando a confiança dos consumidores.

A quinta contribuição é a confirmação da $\mathbf{H}_{\mathbf{3}}$ (a qualidade das informações disponibilizada pelo site de compras on-line influencia positivamente na confiança depositada neste site). Isto revela que as percepções dos consumidores sobre a qualidade das informações são determinantes da confiança no site, reforçando a decisão de concluir uma compra no ambiente on-line. Além disso, ficou evidenciado que a frequência com que os consumidores realizam compras on-line modera a relação entre qualidade das informações disponibilizada pelo site de compras e a confiança que é depositada no referido site. A sexta contribuição se remete à confirmação da $\mathbf{H 5}$ (a confiança dos consumidores influencia positivamente a sua intenção de recompra no site de compras on-line). Evidencia-se que a confiança no site é um elemento essencial para a manutenção da continuidade das relações com os consumidores que realizam suas operações neste ambiente, influenciando positivamente na sua intenção ou atitude de repetir compras.

Quanto às limitações metodológicas, é imperioso ressaltar que um com corte transversal não possibilita a verificação sobre as mudanças na percepção dos consumidores com o passar do tempo. São sugeridos, assim, estudos longitudinais envolvendo os construtos testados. Outra limitação é o fato da pesquisa ter utilizado uma amostra nãoprobabilística. Embora se tenha conseguido uma quantidade razoável de casos válidos, este tipo de amostra constitui uma barreira para a generalização dos resultados. Como o escopo geográfico da pesquisa foi restrito a indivíduos residentes na mesma região (Serra Gaúcha), isto pode ter acarretado algum viés. A realização de pesquisas com amostras probabilísticas mais amplas poderia seria uma forma de atenuar estas duas limitações, podendo trazer resultados diferentes.

Aponta-se a possibilidade de ampliação dos testes de efeito de moderação, verificando os diferentes comportamentos do consumidor entre compras mais ou menos complexas, o risco percebido e o seu nível de envolvimento; bem como análises de mediação tanto com construtos utilizados quanto outros construtos (conveniência, força da marca e preço percebido). Por fim, destaca-se que o Modelo Teórico proposto, mesmo tendo apresentado medidas de ajustes satisfatórias, não deve ter sua validação considerada como definitiva. É oportuno estimular o seu refinamento, replicação e comparação com modelos alternativos (aninhados ou rivais).

\section{Referências}

ASSOCIAÇÃO BRASILEIRA DE COMÉRCIO ELETRÔNICO. Webshoppers 40: e-commerce mantém-se em alto no Brasil. [S.I.]: ABCOMM, 2019.

Disponível em: https://abcomm.org/noticias/webshoppers-40-e-

commerce-mantem-se-em-alta-no-brasil/. Acesso em: 27 set. 2019. 
ABRANTES J.L.; SEABRA, C.; LAGES, C.R.; JAYAWARDHENA, C. Drivers of ingroup and out of group electronic word of mouth (e-WOM). European Journal of Marketing, [s.l.], v. 47, n. 7, p. 1.067-1.088, 2013.

ADNAN, H. An Analysis of the factors affecting online purchasing behavior of Pakistani consumers. International Journal of Marketing Studies, v.6, n. 5, p.133-148, 2014.

ALADWANI, A.M.; PALVIA, P.C. Developing and validating an instrument for measuring user-perceived web quality. Information \& Management, [s.l.], v. 39, n. 6, p. 467-476, 2002.

ALHARBI, I.M.; ZYNGIER, S.; HODKINSON, C. Privacy by design and customers' perceived privacy and security concerns in the success of ecommerce. Journal of Enterprise Information Management, [s.l.], v. 26, n. 6, p.702-718, 2013.

AMA WINTER MARKETING EDUCATORS' CONFERENCE, 2015, San Antonio. Proceedings [...]. San Antonio: AMA Educators Proceedings, 2015.

Disponível em: http://www.ama.org/eventstraining/Conferences/Pages/Winter-Marketing-EducatorsConference.aspx?tab=home. Acesso em: 28 fev. 2015.

ANTONIOU, G.; BATTEN, L. E-commerce: protecting purchaser privacy to enforce trust. Electronic Commerce Research, [s.l.], v. 11, n. 4, p. 421$456,2011$.

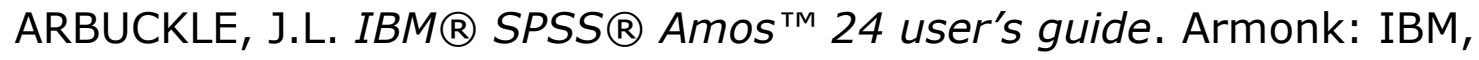
2016.

BAGOZZI, R.P.; PHILLIPS, L.W. Representing and testing organizational theories: a holistic construal. Administrative Science Quarterly, [s.l.], v. 27, n. 3, p. 459-489, 1982.

BAGOZZI, R.P.; YI, Y. Specification, evaluation, and interpretation of structural equation models. Journal of the Academy of Marketing Science, [s.l.], v. 40, n. 1, p. 8-34, 2012.

BAI, B.; LAW, R.; WEN, I. The impact of website quality on customer satisfaction and purchase intentions: evidence from Chinese online visitors. International Journal of Hospitality Management, [s.l.], v. 27, n. 3, p. 391-402, 2008.

BANSAL, G.; ZAHEDI, F. Trust-discount tradeoff in three contexts: frugality moderating privacy and security concerns. Journal of Computer Information Systems, [s.l.], v. 55, n. 1, p. 13-29, 2014.

BARON, R.M.; KENNY, D.A. The moderator-mediator variable distinction in social psychological research: conceptual, strategic, and statistical 
considerations. Journal of Personality and Social Psychology, [s.l.], v. 51, n. 6, p. 1173-1182, 1986.

BART, Y.; SHANKAR, V.; SULTAN, F.; URBAN, G.L. Are the drivers and role of online trust the same for all web sites and consumers? A largescale exploratory empirical study. Journal of Marketing, [s.l.], v. 69, n. 4, p. 133-152, 2005.

BERNARD, E.K.; MAKIENKO, I. The effects of information privacy and online shopping experience in e-commerce. Academy of Marketing Studies Journal, [s.l.], v. 15, n. 1, p. 97-112, 2011.

BIANCHI, C.; ANDREWS, L. Risk, trust, and consumer online purchasing behavior: a Chilean perspective. International Marketing Review, v. 29, n. 3, p. 253-275, 2012.

BOJEI, J.; JULIAN, C.C.; WEL, C.A.B.C.; AHMED, Z.U. The empirical link between relationship marketing tools and consumer retention in retail marketing. Journal of Consumer Behaviour, [s.l.], v. 12, n. 3, p. 171-181, 2013.

BYRNE, B.M. Structural equation modeling with AMOS: basic concepts, applications, and programming. 3rd. ed. New York: Routledge, 2016.

CASALÓ, L.V.; FLAVIÁN, C.; GUINALÍU, M. Antecedents and consequences of consumer participation in on-line communities: the case of the travel sector. International Journal of Electronic Commerce, [s.I.], v. 15, n. 2, p. 137-167, 2010.

CASTAÑEDA, J.A.; MONTOSO, F.J.; LUQUE, T. The dimensionality of customer privacy concern on the internet. Information Review, [s.l.], v. 31, n. 4, p. 420-439, 2007.

CHANG, H.H.; CHEN, S.W. The impact of online store environment cues on purchase intention: trust and perceived risk as a mediator. Information Review, [s.l.], v. 32, n. 6, p. 818-841, 2008.

CHATTERJEE, P. Online reviews: do consumers use them? Advances in Consumer Research, [s.l.], v. 28, n. 1, p. 129-133, 2001.

CHELLAPPA, R.K.; PAVLOU, P.A. Perceived information security, financial liability and consumer trust in electronic commerce transactions. Logistics Information Management, [s.l.], v. 15, n. 5/6, p. 358-368, 2002.

CHEN, J.V.; DAVID, V.C.; RU KUO, C.Y.W.; CAPISTRANO, E.P.S. The antecedents of purchase and re-purchase intentions of online auction consumers. Computers in Human Behavior, [s.l.], v. 54, p. 186-196, 2016. 
CHEUNG, C.M.K.; LEE, M.K.O. What drives consumers to spread electronic word of mouth in online consumer-opinion platforms. Decision Support Systems, [s.l.], v. 53, n. 1, p. 218-225, 2012.

CHEUNG, C.M.K.; THADANI, D.R. The impact of electronic word-of-mouth communication: a literature analysis and integrative model. Decision Support Systems, v. 54, n. 1, p. 461-470, 2012.

CHEVALIER, J.A.; MAYZLIN, D. The effect of word of mouth on sales: online book reviews. Journal of Marketing Research, [s.l.], v. 43, n. 3, p. 345-354, 2006.

CHIU, C.M.; CHANG, C.C.; CHENG, H.L.; FANG, Y.H. Determinants of customer repurchase intention in online shopping. Information Review, [s.l.], v. 33, n. 4, p. 761-784, 2009.

CHIU, C.M.; WANG, E.T.; FANG, Y.H. ; HUANG, H.Y. Understanding customers' repeat purchase intentions in $\mathrm{B} 2 \mathrm{C}$ e-commerce: the roles of utilitarian value, hedonic value and perceived risk. Information Systems Journal, [s.l.], v. 24, n. 1, p. 85-114, 2014.

CHOI, J.; NAZARETH, D. Repairing trust in an e-commerce and security context: an agent-based modeling approach. Information Management \& Computer Security, [s.l.], v. 22, n. 5, p. 490-512, 2014.

CHOU, S; CHEN, C.W.; LIN, J.Y. Female online shoppers: examining the mediating roles of e-satisfaction and e-trust on e-loyalty

development. Internet Research, [s.I.], v. 25, n. 4, p. 542-561, 2015.

$\mathrm{CHU}$, S.C.; KIM, Y. Determinants of consumer engagement in electronic word-of-mouth (e-WOM) in social networking sites. International Journal of Advertising, [s.l.], v. 30, n. 1, p. 47-75, 2011.

CORRITORE, C.L. ; KRACHER, B.; WIEDENBECK, S. On-line trust: concepts, evolving themes, a model. International Journal of HumanComputer Studies, [s.l.], v. 58, n. 6, p. 737-758, 2003.

D'ALESSANDRO, S.; GIRARDI, A.; TIANGSOONGNERN, L. Perceived risk and trust as antecedents of online purchasing behavior in the USA gemstone industry. Asia Pacific Journal of Marketing and Logistics, [s.l.], v. 24, n. 3, p.433-460, 2012.

DEDEKE, A.N. Travel web-site design: information task-fit, service quality and purchase intention. Tourism Management, [s.l.], v. 54, n. 1, p. 541554, 2016. 
DELLAROCAS, C.; AWAD, N.F.; ZHANG, X.M. Exploring the value of online product reviews in forecasting sales: the case of motion pictures. Journal of Interactive Marketing, [s.l.], v. 21, n. 4, p. 23-45, 2007.

DELONE, W. H.; MCLEAN, E. R. The DeLone and McLean model of information systems success: a ten-year update. Journal of Management Information Systems, [s.l.], v. 19, n. 4, p. 9-30, 2003.

DESAI, M.S.; DESAI, K.J.; PHELPS, L.D. E-commerce policies and customer privacy: a longitudinal study (2000-2010). Information Management \& Computer Security, [s.l.], v. 20, n. 3, p. 222-244, 2012.

DINEV, T. Why would we care about privacy? European Journal of Information Systems, [s.l.], v. 23, n. 1, p. 97-102, 2014.

DOMMEYER, C.J.; GROSS, B.L. What consumers know and what they do: an investigation of consumer knowledge, awareness, and use of privacy protection strategies. Journal of Interactive Marketing, [s.l.], v. 17, n. 2, p. 34-51, 2003.

EBITEMPRESA. Balanço 2014 e expectativas para 2015. Disponível em:

http://n.bcash.com.br/blog/2015/02/webshoppers/31 webshoppers Ebit. pdf Acesso em: 26 mar. 2015.

ECOMMERCENEWS. E-commerce brasileiro. Disponível em: https://www.ecommercebrasil.com.br/. Acesso em: 02 mar. 2016.

EGGERT, A. Intangibility and perceived risk in online environments. Journal of Marketing Management, [s.l.], v. 22, n. 5-6, p. 553-572, 2006.

ESCOBAR-RODRÍGUEZ, T.; CARVAJAL-TRUJILLO, E. Online purchasing tickets for low cost carriers: an application of the unified theory of acceptance and use of technology (UTAUT) model. Tourism Management, [s.l.], v. 43, n. 1, p. 70-88, 2014.

ESTEVES, P.S. Uso da internet pelo consumidor da terceira idade: influências do risco percebido e impacto na intenção de compra online. 2014. 270 f. Tese (Doutorado em Administração). Universidade Federal do Rio Grande do Sul, Porto Alegre, 2014.

FANG, Y.; QURESHI, I.; SUN, H.; MCCOLE, P.; RAMSEY, E.; LIM, K.H. Trust, satisfaction, and online repurchase intention: the moderating role of perceived effectiveness of e-commerce institutional mechanisms. MIS Quarterly, [s.l.], v. 38, n. 2, p. 407-427, 2014.

FLAVIÁN, G.; GUINALÍU, M. Consumer trust, perceived security and privacy policy: three basic elements of loyalty to a web site. Industrial Management and Data Systems, [s.l.], v. 106, n. 5, p. 601-620, 2006. 
FORNELL, C.; LARCKER, D. F. Evaluating structural equation models with unobservable variables and measurement error. Journal of Marketing Research, [s.l.], v. 18, n. 1, p.39-50, 1981.

GAO, T.; WANG, Y.; SIRGY, M.J.; BIRD, M.M. An integrative model on the antecedents of buyer decision-making uncertainty in organizational purchasing. Asia-Pacific Advances in Consumer Research, [s.l.], v. 5, n. 1, p. 41-47, 2002.

GARFIELD, M.J.; MCKEOWN, P.G. Planning for internet security. Information Systems Management, [s.l.], v. 14, n. 1, p. 41-46, 1997.

GEFEN, D.; BENBASAT, I.; PAVLOU, P. A research agenda for trust in online environments. Journal of Management Information Systems, [s.I.], v. 24, n. 4, p. 275-286, 2008.

GUO, M.A. Comparative study on consumer right to privacy in ecommerce. Modern Economy, [s.l.], v. 3, n. 4, p. 402-407, 2012.

GUPTA, S.; KIM, H.W. The moderating effect of transaction experience on the decision calculus in on-line repurchase. International Journal of Electronic Commerce, [s.l.], v. 12, n. 1, p. 127-158, 2007.

HA, H.Y.; JANDA, S.; MUTHALY, S.K. A new understanding of satisfaction model in e-re-purchase situation. European Journal of Marketing, [s.l.], v. 44, n. 7/8, p. 997-1016, 2010.

HAIR, Jr., J.F.; BLACK, W.C.; BABIN, B.J.; ANDERSON, R.E.; TATHAM, R.L. 7th ed. Multivariate data analysis. New Jersey: Pearson, 2010.

HENNIG-THURAU, T.; GWINNER, K.P.; WALSH, G.; GREMLER, D.D. Electronic word-of-mouth via consumer-opinion platforms: what motivates consumers to articulate themselves on the internet? Journal of Interactive Marketing, [s.l.], v. 18, n. 1, p. 38-52, 2004.

HSIAO, K.L.; LIN, J.C.C.; WANG, X.Y.; LU, H.P.; YU, H. Antecedents and consequences of trust in online product recommendations: an empirical study in social shopping. Information Review, [s.l.], v. 34, n. 6, p. 935953, 2010.

HSU, M.; CHANG, C.; CHUANG, L. Understanding the determinants of online repeat purchase intention and moderating role of habit: the case of online group-buying in Taiwan. International Journal of Information Management, [s.l.], v. 35, n. 1, p. 45-56, 2015.

HU, X.; HA, L. Which form of word-of-mouth is more important to online shoppers? A comparative study of WOM use between general population and college students. Journal of Communication and Media Research, [s.l.], v. 7, n. 2, p. 15-35, 2015. 
HUANG, L. Exploring the determinants of e-loyalty among travel agencies. The Service Industries Journal, [s.I.], v. 28, n. 2, p. 239-254, 2008.

JOIA, L.A.; OLIVEIRA, L.C.B. Development and testing of an e-commerce website evaluation model. Revista de Administração Mackenzie, [s.l.], v. 9, n. 1, p. 11-36, 2008.

KAYA, T. Multi-attribute evaluation of website quality in e-business using an integrated fuzzy AHPTOPSIS methodology. International Journal of Computational Intelligence Systems, [s.I.], v. 3, n. 3, p. 301-314, 2010.

KHALIFA, M.; LIU, V. Online consumer retention: contingent effects of online shopping habit and online shopping experience. European Journal of Information Systems, [s.l.], v. 16, n. 6, p. 780-792, 2007.

KIM, D.J.; FERRIN, D.L.; RAO, H.R. A trust-based consumer decisionmaking model in electronic commerce: the role of trust, perceived risk, and their antecedents. Decision Support Systems, [s.I.], v. 44, n. 2, p. 544-564, 2008.

KIM, S.; NOH, M.J. Determinants influencing consumers' trust and trust performance of social commerce and moderating effect of experience. Information Technology Journal, [s.l.], v. 11, n. 10, p. 1369$1380,2012$.

KLINE, R.B. Principles and practice of structural equation modeling. 3rd ed. New York: Guilford Press, 2011.

KUAN, H.H.; BOCK, G.W.; VATHANOPHAS, V. Comparing the effects of website quality on customer initial purchase and continued purchase at ecommerce websites. Behaviour \& Information Technology, [s.I.], v. 27, n. 1, p. 3-16, 2008.

LEE, K.C.; KANG, I. ; MCKNIGHT, D.H. Transfer from offline trust to key online perceptions: an empirical study. IEEE Transactions on Engineering Management, [s.l.], v. 54, n. 4, p. 729-741, 2007.

LIN, M.J.; WANG, W.T. Explaining online customer repurchase intentions from a relationship-marketing perspective: an integration of the 4Rs marketing strategy and customer trust. Journal of Organizational and End User Computing, [s.l.], v. 27, n. 3, p. 1-26, 2015.

LIU, C.; MARCHEWKA, J.T.; LU, J.; YU, C.S. Beyond concern: a privacytrust-behavioral intention model of electronic commerce. Information \& Management, [s.l.], v. 42, n. 2, p. 289-304, 2005.

LÓPEZ, M.; SICILIA, M. Determinants of e-WOM influence: the role of consumers internet experience. Journal of Theoretical and Applied Electronic Commerce Research, [s.l.], v. 9, n. 1, p. 28-43, 2014. 
LU, Y.; LU, Y.; WANG, B. Effects of dissatisfaction on customer repurchase decisions in e-commerce: an emotion-based perspective. Journal of Electronic Commerce Research, [s.l.], v. 13, n. 3, p. 224-237, 2012.

MALHOTRA, N.K.; BIRKS, D. ; WILLS, P. Marketing research: applied approach. 4th ed. New York: Pearson, 2012.

MCKINNEY, V.; YOON, K.; ZAHEDI, F.M. The measurement of webcustomer satisfaction: an expectation and disconfirmation approach. Information Systems Research, [s.l.], v. 13, n. 3, p. 296-315, 2002.

MCKNIGHT, D.H. ; KACMAR, C.J.; CHOUDHURY, V. Dispositional trust and distrust distinctions in predicting high-and low-risk internet expert advice site perceptions. E-Service, [s.I.], v. 3, n. 2, p. 35-58, 2004.

METZGER, M.J. Privacy, trust, and disclosure: exploring barriers to electronic commerce. Journal of Computer-Mediated Communication, [s.l.], v. 9, n. 4, p. 1-18, 2004.

MEZIANE, F.; KASIRAN, M.K. Evaluating trust in electronic commerce: a study based on the information provided on merchants' websites. Journal of the Operational Research Society, [s.l.], v. 59, n. 4, p. 464-472, 2008.

MSI - MARKETING SCIENCE INSTITUTE. 2014-2016 Research priorities. Disponível em: https://www.msi.org/uploads/files/MSI RP14-16.pdf. Acesso em: 22 dez. 2015.

NUNKOO, R.; RAMKISSOON, H.; GURSOY, D. Use of structural equation modeling in tourism research: past, present, and future. Journal of Travel Research, [s.l.], v. 52, n. 6, p. 759-771, 2013.

PANDA, S.K.; PANDA, J.K. Understanding the impact of perceived security on consumer trust in electronic banking. Research Expo International Multidisciplinary Research Journal, [s.l.], v. 2, n. 4, p. 112-120, 2012.

PARK, J.; LEE, Y.; WIDDOWS, R. Empirical investigation on reputation and product information for trust formation in consumer to consumer market. Journal of the Academy of Business and Economics, [s.l.], v. 3, n. 1, p. 231-239, 2004.

PETERSON, R.A.; BALASUBRAMANIAN, S.; BRONNENBERG, B. J. Exploring the implications of the Internet for consumer marketing. Journal of the Academy of Marketing Science, [s.I.], v. 25, n. 4, p. 329-346, 1997.

PONTE, E.B.; CARVAJAL-TRUJILLO, E.; ESCOBAR-RODRÍGUEZ, T. Influence of trust and perceived value on the intention to purchase travel online: integrating the effects of assurance on trust antecedents. Tourism Management, [s.l.], v. 47, n. 1, p. 286-302, 2015. 
QURESHI, I.F.; RAMSEY, E.; MCCOLE, P.; IBBOTSON, P.; COMPEAU, D. Understanding online customer repurchasing intention and the mediating role of trust: an empirical investigation in two developed

countries. European Journal of Information Systems, [s.l.], v. 18, n. 3, p. 205-222, 2009.

RAFFAELE, A.; ORLANDO, T. Customer loyalty: an empirical study on Italian e-commerce websites. Chinese Business Review, [s.l.], v. 13, n. 6, p. 388-398, 2014.

RAY, S.; OW, T.; KIM, S. Security assurance: how online service providers can influence security control perceptions and gain trust. Decision Sciences Journal, [s.l.], v. 42, n. 2, p. 391-412, 2011.

REHMAN, S. Influence of e-commerce and its emerging innovations in banks. International Journal of Marketing and Technology, [s.l.], v. 2, n. 8, p. 289-303, 2012.

ROCA, J.C.; GARCÍA, J.J.; VEGA, J.J. The importance of perceived trust, security and privacy in online trading systems. Information Management and Computer Security, [s.l.], v. 17, n. 2, p. 96-113, 2009.

ROMAN, S. The ethics of online retailing: a scale development and validation from the consumers' perspective. Journal of Business Ethics, [s.l.], v. 72, n. 2, p. 131-148, 2007.

SALISBURY W.D.; PEARSON, R.A.; PEARSON, A.W.; MILLER, D.W. Perceived security and world wide web purchase intention. Industrial Management \& Data Systems, [s.l.], v. 101, n. 4, p. 165-177, 2001.

SCHLOSSER, A.E.; WHITE, T.B.; LLOYD, S.M. Converting website visitors into buyers: how website investment increases consumer trusting beliefs and online purchase intentions. Journal of Marketing, [s.l.], v. 70, n. 2, p. 133-148, 2006.

SCHÜTZE, N. Electronic word-of-mouth communication for local service providers. Technology Innovation Management Review, [s.l.], v. 4, n. 4, p. 35-42, 2014.

SHCHIGLIK, C.; BARNES, S. Evaluating website quality in the airline industry. Journal of Computer Information Systems, [s.l.], v. 44, n. 3, p. $17-25,2004$.

SHEPPARD, B.H.; SHERMAN, D.M. The grammars of trust: a model and general implications. Academy of Management Review, [s.l.], v. 23, n. 3, p. 422-437, 1998.

SHIN, J.I.; CHUNG, K.H.; OH, J.S.; LEE, C.W. The effect of site quality on repurchase intention in Internet shopping through mediating variables: 
the case of university students in South Korea. International Journal of Information Management, [s.I.], v. 33, n. 3, p. 453-463, 2013.

SRINIVASAN, S.S.; ANDERSON, R.; PONNAVOLU, K. Customer loyalty in e-commerce: an exploration of its antecedents and consequences. Journal of Retailing, [s.l.], v. 78, n. 1, p. 41-50, 2002.

STATISTA. B2C e-commerce sales worldwide from 2012 to 2018. [S.I.]: STATISTA, [2015?]. Disponível em:

http://www.statista.com/statistics/261245/b2c-e-commerce-salesworldwide/. Acesso em: 03 abr. 2016.

STAUSS, B. Global word of mouth: service bashing on the internet is a thorny issue. Marketing Management, [s.l.], v. 6, n. 3, p. 28-30, 1997.

TEHREEM, A. Factors deriving consumers' repurchase intention in online shopping: a Pakistani consumer's perspective. International Journal of Management Sciences and Business Research, [s.l.], v. 5, n. 12, p. 261270, 2017.

TENG, S.; KHONG, K.W.; GOH, W.W.; CHONG, A.Y.L. Examining the antecedents of persuasive e-WOM messages in social media. Information Review, [s.l.], v. 38, n. 6, p. 746-768, 2014.

TRUSOV, M.; BUCKLIN, R.E.; PAUWELS, K. Effects of word-of-mouth versus traditional marketing: findings from an internet social networking site. Journal of Marketing, [s.l.], v. 73, n. 5, p. 90-102, 2009.

TSAO, W.Y. The fitness of product information: evidence from online recommendations. International Journal of Information Management, $v$. 33, n. 1, p. 1-9, 2013.

URBAN, G.L. ; AMYX, C. ; LORENZON, A. Online trust: state of the art, new frontiers, and research potential. Journal of Interactive Marketing, [s.l.], v. 23, n. 2, p. 179-190, 2009.

VAITHIANATHAN, S. A review of e-commerce literature on India and research agenda for the future. Electronic Commerce Research, [s.l.], v. 10 , n. 1, p. 83-97, 2010.

VALLEJO, J.M.; REDONDO, Y.P.; ACERETE, A. U. Las características del boca-oído electrónico y su influencia en la intención de recompra online. Revista Europea de Dirección y Economía de la Empresa, [s.l.], v. 24, n. 2, p. 61-75, 2015.

VAN SLYKE, C.; SHIM, J.T.; JOHNSON, R.; JIANG, J.J. Concern for information privacy and online consumer purchasing. Journal of the Association for Information Systems, [s.l.], v. 7, n. 6, p. 415-444, 2006. 
VENKATESH, A. Cybermarketscapes and consumer freedoms and identities. European Journal of Marketing, [s.l.], v. 32, n. 7/8, p. 664-676, 1998.

WANG, Y.D.; EMURIAN, H.H. An overview of online trust: concepts, elements, and implications. Computers in Human Behavior, [s.l.], v. 21, n. 1 , p. 105-125, 2005.

YOO, C.W.; SANDERS, G.L.; MOON, J. Exploring the effect of e-WOM participation on e-loyalty in e-commerce. Decision Support Systems, [s.l.], v. 55, n. 3, p. 669-678, 2013. 